\title{
Transcriptional control of alternative splicing along time: Ideas change, experiments remain
}

\author{
ALBERTO R. KORNBLIHTT \\ Instituto de Fisiología, Biología Molecular y Neurociencias (IFIBYNE-UBA-CONICET) and Departamento de Fisiología, Biología \\ Molecular y Celular, Facultad de Ciencias Exactas y Naturales, Universidad de Buenos Aires, Ciudad Universitaria, Pabellón 2, \\ C1428EHA Buenos Aires, Argentina
}

Evidence on the co-transcriptionality of splicing and on a role for the transcription machinery on splice site selection started to be published before the launching of RNA. However, it was during the last 20 years that initial suspicion and speculation gave room to a profuse body of evidence supporting a radical change of view on the regulatory mechanisms of splicing, originally conceived as a purely post-transcriptional event. Surprisingly, the first evidence for co-transcriptional splicing is still one of the strongest ones, probably because "seeing is believing": In 1988 Beyer and Osheim performed cytological examination of Drosophila genes caught in active transcription and produced beautiful and compelling EM images revealing that many introns are excised as the mRNA is being synthesized, before RNA polymerase II (RNAPII) reaches the end of the gene. In agreement with this observation, a few years later, using light microscopy, Jeanne Lawrence confirmed that pre-mRNAs are constrained from free diffusion and that splicing appeared to occur within small tracks in the vicinity of gene loci. This idea would be reinforced by similar but not identical observations from David Spector's lab showing that splicing factors are recruited from speckles to the sites of transcription upon transcriptional activation. However, back to the Beyer and Osheim observation, if splicing was co-transcriptional, could it also be mechanistically coupled to transcription? In April 1993, Arno Greenleaf published an "Open Question" article in TiBS with a speculative model in which positively charged splicing factors could be tethered to the phosphorylated, and therefore negatively charged, carboxy-terminal domain (CTD) of elongating RNAPII, which would then help splicing factors to gain access to their binding sites in the nascent pre-mRNA. This provocative idea proved to be extremely fruitful to investigate CTD functions other than transcription and opened the whole field of coupling. Indeed, in the following years, a physical association of the highly phosphorylated form of RNAPII large subunit with splicing factors and spliceosome

Corresponding author: ark@fbmc.fcen.uba.ar

Article and publication date are at http://www.rnajournal.org/cgi/doi/ 10.1261/rna.051151.115. Freely available online through the RNA Open Access option. components as well as a role in stimulating splicing was demonstrated by the Corden, Berezney, and Manley labs. These in vitro experiments were paralleled by an in vivo proof provided by a seminal Nature paper of 1997 from the Bentley lab showing that when transcription in cultured cells is performed by an RNAPII bearing a truncated CTD, RNA splicing, $3^{\prime}$ end processing, and transcriptional termination are affected. Bentley proposed then the concept of "mRNA factory," suggesting that RNAPII and its associated factors not only perform transcription but are also key for the co-transcriptional covalent modifications of the pre-mRNA. The CTD being a particular feature of RNAPII, these findings were highly consistent with observations of the mid-' $80 \mathrm{~s}$ that RNA processing was less efficient when intron-bearing genes were put under the control of RNAPI or RNAPIII promoters, which would be later complemented by the Rosbash lab extending similar observations to T7 phage promoters. At present there is some controversy as to whether splicing factors interact directly with RNAPII. Using reporter genes embedded in bacterial artificial chromosomes to guarantee chromatin environments of the stably transfected constructs similar to those of the corresponding endogenous genes, Karla Neugebauer showed in 2009 that all SR protein interactions with RNAPII are RNase-sensitive, suggesting that SR proteins are not preassembled with the enzyme but recruited to nascent mRNA.

There is abundant literature on the coupling of transcription with capping and $3^{\prime}$ end formation. However, because of space restrictions, I will focus on the coupling with splicing, and in particular, with alternative splicing. In 1995 I proposed a new graduate student in the lab, Paula Cramer, to explore if changing the RNAPII promoter of gene by the RNAPII promoter of another gene would affect the patterns of alternative splicing of the produced transcript. If the experiment works, I said, you will have a thesis and a paper. If promoter swapping does not affect alternative splicing, you may have a thesis, but not papers. Paula got both thesis and

(C) 2015 Kornblihtt This article, published in RNA, is available under a Creative Commons License (Attribution-NonCommercial 4.0 International), as described at http://creativecommons.org/licenses/by-nc/4.0/. 
papers. She showed, in papers published in collaboration with Tito Baralle in 1997 and with Javier Cáceres in 1999, that changing, for instance, the alpha globin promoter by the fibronectin promoter in the same transcriptional unit containing an alternative splicing event caused a 10-fold increase in the inclusion levels of the alternative cassette into the mature mRNA. It was clear to us that promoter swapping was a tool, an artificial way to demonstrate that transcription and alternative splicing are coupled. A more physiological scenario should test differential occupation of the same promoter by distinct transcription factors. Accordingly, we found later that mutating or inserting enhancers, as well as directing two different transcription factors to a single promoter, had profound effects on the elicited alternative splicing patterns. To our relief, the promoter effect was not an oddity of our lab: Didier Aubeouf, working in Bert O'Malley and Sue Berget's labs, soon found that steroid hormone nuclear receptors and co-activators affected alternative splicing in a promoter-dependent manner and the Blencowe lab reported that transcriptional activators modulate constitutive splicing in a CTD-dependent manner.

To further investigate the mechanisms of coupling we envisioned two nonexclusive working models: Alternative splicing is affected by the recruitment of splicing factors to the transcription apparatus (recruitment coupling) or by the speed of RNAPII elongation (kinetic coupling). Again due to space limitations I will only focus on kinetic coupling for which we published supporting indirect evidence in 2001 and more direct and conclusive data in collaboration with David Bentley and Claudio Alonso in 2003, with Manuel de la Mata as first author: Transcription by a slow mutant of RNAPII promoted higher exon inclusion of a transcript encoded by a reporter minigene but also affected alternative splicing of the Drosophila endogenous Ubx transcript. A kinetic role for transcription on splicing had been suggested before by the Eperon lab in 1988, by the Nick Proudfoot/Chris Smith labs a decade later, and confirmed in an artificially created alternative splicing event in yeast by Manny Ares in 2003. For some years we interpreted the effects of slow elongation in the light of the "first come, first served" mechanism, assuming that for the upstream intron of an alternative cassette exon, "first served" meant "first excised." In other words, a slow RNAPII would favor the usage of a weak $3^{\prime}$ splice site of the upstream intron in detriment of the stronger $3^{\prime}$ splice site of the downstream intron. However, more recently, we and others realized that slow elongation favors recruitment of splicing factors to the weak $3^{\prime}$ splice site of the upstream intron and that the actual catalytic step that excises the upstream intron may occur later, even after transcription was completed. We interpret now "first served" as "first committed" to splice, independently of whether the catalysis takes place co- or post-transcriptionally. Another addition to the original model is that slow elongation can also promote skipping of certain alternative exons by favoring recruitment of negative splicing factors to their target sites in the upstream intron, as we showed in last year's Mol Cell paper together with Gwendal Dujardin and Laurent Corcos.

The influence of RNAPII elongation on alternative splicing was confirmed genome-wide by the Blencowe and Bentley labs. Interestingly, in a recent Genes Dev paper, Bentley used both slow and fast RNAPII mutants and confirmed that slow elongation promotes inclusion or skipping depending on the kind of alternative exon. They found many alternative cassette exons that, as expected, respond in opposite ways to the slow and fast mutants, but they also found a subset of exons that respond in the same direction whether elongation is faster or lower than normal. This led them to propose that, within a narrow range, proper elongation speed is critical for adequate exon inclusion, which in my opinion complements but does not replace the "first come, first serve" model. Indeed this model is also supported by experiments of a different nature that analyze the links between intragenic pauses and splicing and alternative splicing, published by the groups of Nick Proudfoot, Jean Beggs, Karla Neugebauer, Christian Muchardt, Mariano García-Blanco, Shalini Oberdoerffer, and myself. Independently of the interpretation model, evidence for the modulation of alternative splicing by elongation has accumulated, from different labs, experimental systems and methodologies that include not only mammalian cells but also plants, as shown in a report published by Świeżewski and colleagues this year. Nevertheless, a role for elongation is not exempt from criticism: In a comprehensive review published in RNA two years ago, Jim Darnell argues that since in mammalian cells splicing requires several minutes after synthesis of the $3^{\prime}$ intron-exon junction, brief pausing (seconds) of an RNAPII that is running at 3-4 $\mathrm{kb} / \mathrm{min}$ would have no significant effects on the splicing outcome. I think that this might be true if splicing catalysis but not splicing commitment, as explained above, is the step affected by elongation.

Changes in elongation can be elicited by changes in RNAPII intrinsic activity through post-translational modifications (e.g., CTD phosphorylation) and/or association of RNAPII to regulatory factors. Alternatively, elongation can be modulated by changes in chromatin structure. An example of the first mechanism is the regulation of alternative splicing by DNA damage caused by UV irradiation that our lab reported in a 2009 Cell paper with Manuel Muñoz as first author, extended genome-wide by the Blencowe lab in 2011. As for the roles of histone marks and chromatin structure on splicing, a whole fascinating chapter of RNA biology is being written. The idea that chromatin structure could affect alternative splicing was suggested by experiments showing that inclusion of a cassette exon in a mature mRNA was increased in response to replication of the template DNA performed by Sebastián Kadener when he was a graduate student in my lab and published in 2001: The more compact chromatin structure of the replicated reporter plasmid acted as a barrier to elongation resulting in higher inclusion. The fact that the effect of template replication on alternative splicing 
was reverted by chromatin relaxing agents supported our interpretation. A detailed description of the multiple contributions exceeds the limits of this article. Pioneer evidence showing that chromatin remodelers such as SWI/SNF control alternative splicing was published by Batsché, Muchardt, and colleagues in 2006. Since then, splicing and/or alternative splicing regulation associated to the deposition of specific histone marks was revealed by several groups, including those of Danny Reinberg, Julie Ahringer, Tom Misteli, and myself, through the works of Ignacio Schor and Mariano Alló. The S. Oberdoerffer lab also showed in 2011 that DNA methylation and CTCF binding regulate alternative splicing through kinetic coupling. The finding that nucleosomes are preferentially positioned in exons (reported almost simultaneously in 2009 by the groups of Gil Ast, Roderic Guigó/ Juan Valcárcel, J. Komorowski, Chris Burge, and John Mattick) as well as the unexpected roles of noncoding RNAs and argonaute proteins in the control of alternative splicing in the nucleus, reported by our group in 2009 and 2014, and by the Annick Harel Bellan/Eric Batsché groups in 2012, add additional layers of complexity, whose biological implications and mechanistic details are still poorly understood and will be hopefully revealed before the 40th anniversary of the RNA journal.

\section{Acknowledgments}

I wish to apologize to the many colleagues whose relevant work could not be commented on due to space restrictions. A fully referenced version can be requested to ark@fbmc.fcen.uba.ar. This work was supported by the Agencia Nacional de Promoción de Ciencia y Tecnología of Argentina, the Universidad de Buenos Aires, and the Howard Hughes Medical Institute. A.R.K. is a career investigator of the Consejo Nacional de Investigaciones Científicas y Técnicas of Argentina (CONICET) and an HHMI Senior International Research Scholar. 

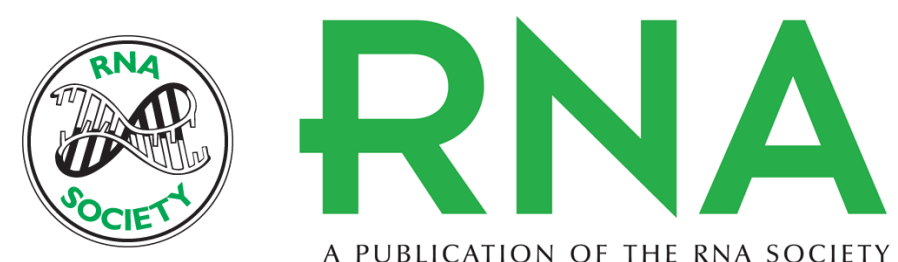

A PUBLICATION OF THE RNA SOCIETY

\section{Transcriptional control of alternative splicing along time: Ideas change, experiments remain}

Alberto R. Kornblihtt

RNA 2015 21: 670-672

Open Access Freely available online through the RNA Open Access option.

Creative This article, published in RNA, is available under a Creative Commons License

Commons (Attribution-NonCommercial 4.0 International), as described at

License http://creativecommons.org/licenses/by-nc/4.0/.

Email Alerting Receive free email alerts when new articles cite this article - sign up in the box at the

Service top right corner of the article or click here.

To subscribe to $R N A$ go to:

http://rnajournal.cshlp.org/subscriptions

(C) 2015 Kornblihtt; Published by Cold Spring Harbor Laboratory Press for the RNA Society 